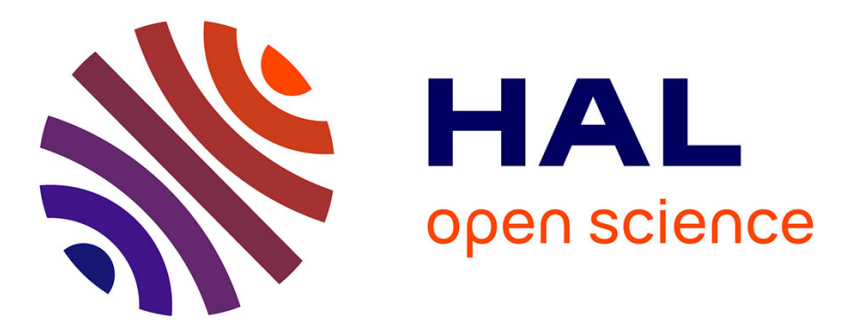

\title{
Hybrid kinematic model applied to the under-actuated robotic hand Prosthesis ProMain-I and experimental evaluation
}

J. Ramirez, A. Rubiano, N. Jouandeau, M N El Korso, L. Gallimard, O. Polit

\section{- To cite this version:}

J. Ramirez, A. Rubiano, N. Jouandeau, M N El Korso, L. Gallimard, et al.. Hybrid kinematic model applied to the under-actuated robotic hand Prosthesis ProMain-I and experimental evaluation. 14th IEEE/RAS-EMBS International Conference in reabilitation robotics, Aug 2015, Singapour, Singapore. 10.1109/ICORR.2015.7281216 . hal-02317212

\section{HAL Id: hal-02317212 \\ https://hal.science/hal-02317212}

Submitted on 15 Oct 2019

HAL is a multi-disciplinary open access archive for the deposit and dissemination of scientific research documents, whether they are published or not. The documents may come from teaching and research institutions in France or abroad, or from public or private research centers.
L'archive ouverte pluridisciplinaire HAL, est destinée au dépôt et à la diffusion de documents scientifiques de niveau recherche, publiés ou non, émanant des établissements d'enseignement et de recherche français ou étrangers, des laboratoires publics ou privés. 


\title{
Hybrid kinematic model applied to the under-actuated robotic hand Prosthesis ProMain-I and experimental evaluation
}

\author{
J. L. Ramírez ${ }^{1}$, A. Rubiano ${ }^{1,3}$, N. Jouandeau ${ }^{2}$, M. N. El Korso ${ }^{1}$, L. Gallimard ${ }^{1}$, O. Polit $^{1}$
}

\begin{abstract}
Upon the appearance of nylons, silicon, tendons, smart and soft materials, wearable robotics is going closer to the human body, leading robotics and biomechanics to provide us new physical rehabilitations and improvements. In the area that concerns soft robotic prosthesis, the main challenge is the design of well sized mechatronic limbs and smart controllers that should help people to achieve desired movements. As a consequence, we present a hybrid model that allows different ways of representing hand poses, according to special interactions that arise from soft robotics chains. Our hybrid model uses the positions of finger's parts computed with the Denavit-Hartenberg (DH) method mixed with the quaternions representation to avoid singularities and to reduce the number of DH parameters. Kinematic and dynamic of finger motions are evaluated using an experimental setup with mechanical parts produced by $3 \mathrm{D}$ printing and different actuators. Finally, experimental results are compared with the theoretical values and demonstrate the accuracy of our model.
\end{abstract}

\section{INTRODUCTION}

Considering that the aim of wearable robotics is to improve or give back physical skills, one of the greatest challenges is to provide a safe, dexterous, and easy to use robotic system. Therefore, the growing interest in the design of robotic hand prostheses able to achieve human manipulation skills.

The task of designing or improving a robotic hand (to replicate the grasping capabilities and the kinematic function of the human hand) involves the consideration of a high complexity sensory and motor functions. Some examples of robotic hands that could be used as a prosthesis are available in the literature [1]. These robotic hands could be classified as commercial (e.g i-Limb) or research purposes (e.g DLRHIT II or UB Hand 4) [2]. Even so, the actual state-of-the-art has not fulfilled the requirements of dexterous manipulation, in terms of physical, actuation, and kinematic properties [3].

Recently, two important considerations have been proposed to increase the performance and the simplicity of robotic hand prostheses. Such as the postural synergies [4] and the adaptive synergies [5]. These synergies are used to formulate grasping forces [6]. Adaptive synergies are particularly referred to the consideration of variable stiffness joints in soft robots, interacting in an adaptive way with the environment and the objects to grasp.

\footnotetext{
*This work was supported by Université Paris Lumière

${ }^{1}$ LEME, Université Paris Ouest Nanterre La Défense, 50, rue de Sèvres, 92410 Ville d'Avray-FR j1.ramirez_arias du-paris10.fr

${ }^{2}$ LIASD, Université Paris 8, 2, Rue de la Liberté, 93526 Saint-Denis-FR

${ }^{3}$ A. Rubiano is Ph.D. student at Université Paris Ouest Nanterre La Défense and is with the Universidad Militar Nueva Granada-CO
}

These approaches allow the design of underactuated robotic hands to accomplish a soft-synergy model, which provides a robust and compliance mechanism [5]. Despite the excellent results to control the internal forces needed to hold an object, the variable stiffness joints state a challenge related to the modeling and handling of not previously considered movements.

Consequently, the synergies approach has been combined with the parameterization of Denavit- Hartenberg (DH) [4]. The DH parameters and its variations were proposed for rigid robots, and new developments are necessary to calculate the kinematic and the dynamic of soft robots.

The main drawback of the DH methods is the limitation of active rotations because they are only possible around $z$ axes of each joint. Thus, a new framework must be added if an extra rotation is needed. Furthermore, the use of homogeneous matrices could cause singularities due to the presence of not considered rotations introduced with soft materials.

An interesting approach of kinematic modelization is presented in [7], where the author proposes a model based on DH parameterization and unit quaternion instead of homogeneous matrices. In the same way, [8] presented a methodology for the geometric design of $3 \mathrm{R}$ manipulators using the $\mathrm{DH}$ and quaternions. The $\mathrm{DH}$ parameterization and the representation of rotations using quaternions were tested in [9] to express deformation in 3D CAD models. Even so, these methods introduce a real difficulty because they remove totally the homogeneous matrices, and a modelling using only quaternions is not efficient from a computational point of view [10].

For these reasons, we present in this paper a new hybrid model that improves the representation of rotations that arise from soft robotics prosthesis movements. Our model combines the parameterization of $\mathrm{DH}$ method with quaternions to formulate soft rotations. This new method allows to: avoid the singularities, reduce the number of rotation parameters and unify the formulation to describe the kinematic of a soft robot.

Furthermore, we propose two new experimental setup: i) the first one is carried out to evaluate the performance of a first prototype of a robotic finger for the task of precision grasping, ii) the second one permits to compare two actuation mechanisms and five different actuators using the same finger. Finally, the results of these experiments are compared with the theoretical ones obtained from our model. 


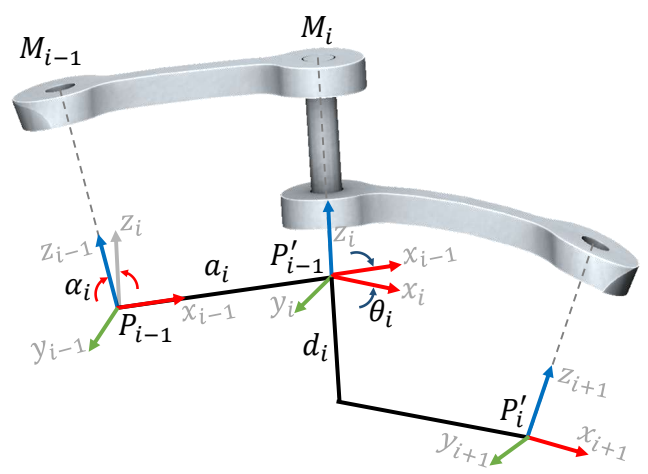

Fig. 1: Graphical representation of DHKK parameters

\section{DenhaVit-Hartenberg Parameterization ModifiEd By KHALIL AND KLEINFINGER, MiXed With QUATERNIONS FORMULATION}

In order to propose a performant method to model the kinematic of a soft robot, we propose a hybrid method, that consist in the utilization of the Denhavit-Hartenberg parameterization Modified by Khalil and Kleinfinger (DHKK), and the formulation of soft rotations using quaternions.

For the purpose of formulating the unexpected rotations that arise from soft robotics movements, our hybrid method represents the kinematic, using DHKK and homogeneous matrices for rigid rotations, and introduces the formulation of soft rotations using quaternions (SRQ). The SRQ is launched taking into account several parameters as the desired movement precision, the direction of movement vectors, and the stiffness of the joints. Likewise, the SRQ can be launched by sensory feedback.

\section{A. Denhavit-Hartenberg parameterization Modified by Khalil And Kleinfinger}

The DHKK convention, allows the representation of openloop and close-loop kinematic chains, and presents a convenient definition of the axis $\vec{z}_{i}$, which corresponds to the rotation axe of the $i-t h$ joint. The angle of rotation around $\vec{z}_{i}$ is denoted by $\theta_{i}$, and is applied using a transformation matrix, that is described in (1), which result of the application of: a rotation $\alpha_{i}$ around $\vec{x}_{i-1}$, a translation $a_{i}$ along of $\vec{x}_{i-1}$, a rotation $\theta_{i}$ around $\vec{z}_{i}$, and a translation $d_{i}$ along of $\vec{z}_{i}$ [9].

$$
{ }^{i-1} T_{i}=\left[\begin{array}{cccc}
\cos \theta_{i} & \sin \theta_{i} & 0 & a_{i} \\
\sin \theta_{i} \cos \alpha_{i} & \cos \theta_{i} \cos \alpha_{i} & \sin \alpha_{i} & \sin \alpha_{i} d_{i} \\
\sin \theta_{i} \sin \alpha_{i} & \cos \theta_{i} \sin \alpha_{i} & \cos \alpha_{i} & \cos \alpha_{i} d_{i} \\
0 & 0 & 0 & 1
\end{array}\right]
$$

The parameters $\alpha_{i}, a_{i}, \theta_{i}$ and $d_{i}$, are known as the DHKK parameters, a graphical representation of the parameters is shown in Fig. 1. Usually, the task of calculation of DHKK parameters is hard, because is performed manually, and is more difficult when there are multiple kinematic chains. Even that, a method to automatically generate these parameters is presented in [11], and is the adopted methodology to parameterize in the present work.

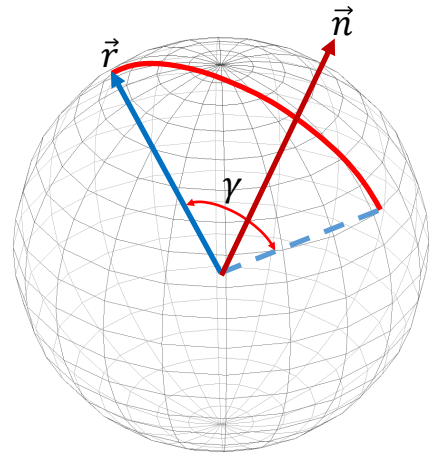

Fig. 2: Rotation $\gamma$ applied to $\vec{r}$ around $\vec{n}$

Consequently, the kinematic of a robot composed of $n$ joints is:

$$
{ }^{0} T_{n}=\prod_{i=1}^{n}{ }^{i-1} T_{i}
$$

As result the matrix ${ }^{0} T_{n}$ is a composition of the orientation of the end effector ${ }^{0} R_{n}$, and the position vector $\left[{ }^{0} P_{n}^{x},{ }^{0} P_{n}^{y},{ }^{0} P_{n}^{z}\right]^{T}$, as shown in the following expression:

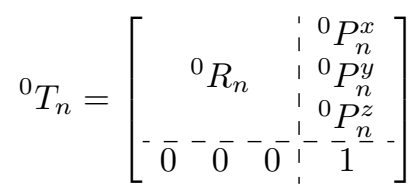

\section{B. Formulation of Rotations Using Quaternions}

The quaternion [12] is a composition of four coefficients, as shown in (4), usually represented with ordered pairs [13] as $\mathbb{H}=\llbracket h_{0}, \vec{h} \rrbracket$, where $\vec{h}=\left(h_{1}, h_{2}, h_{3}\right)$.

$$
\mathbb{H}=h_{0}+h_{1} i+h_{2} j+h_{3} k
$$

The components $i, j$, and $k$ represent the unit vectors that match the direction of $\vec{h}$, and satisfy:

$$
i^{2}=j^{2}=k^{2}=i j k=-1
$$

The real number $h_{0}$ represented as quaternion, is denoted by $\mathbb{H}_{0}=\llbracket h_{0}, \overrightarrow{0} \rrbracket$ and the pure quaternion of the vector $\vec{p}$ is given by $\mathbb{P}=\llbracket 0, \vec{p} \rrbracket$, whose conjugate is $\overline{\mathbb{P}}=\llbracket 0,-\vec{p} \rrbracket$. As presented in [14], two quaternions $\mathbb{H}=\llbracket h_{0}, \vec{h} \rrbracket$ and $\mathbb{V}=$ $\llbracket v_{0}, \vec{v} \rrbracket$ can be multiplied as:

$$
\mathbb{H} \mathbb{V}=\llbracket h_{0} v_{0}-\vec{h} \cdot \vec{v}, h_{0} \vec{v}+v_{0} \vec{h}+\vec{h} \times \vec{v} \rrbracket
$$

In the 3D space, a rotation $\gamma$ applied to the vector $\vec{r}$ around the vector $\vec{n}$, shown in Fig. 2, can be expressed using (6) as:

\section{$\mathbb{N R} \overline{\mathbb{N}}$}

where $\mathbb{N}=\llbracket \cos (\gamma / 2), \vec{n} \sin (\gamma / 2) \rrbracket$

$$
\begin{aligned}
& \mathbb{R}=\llbracket 0, \vec{r} \rrbracket \\
& \overline{\mathbb{N}}=\llbracket \cos (\gamma / 2),-\vec{n} \sin (\gamma / 2) \rrbracket
\end{aligned}
$$




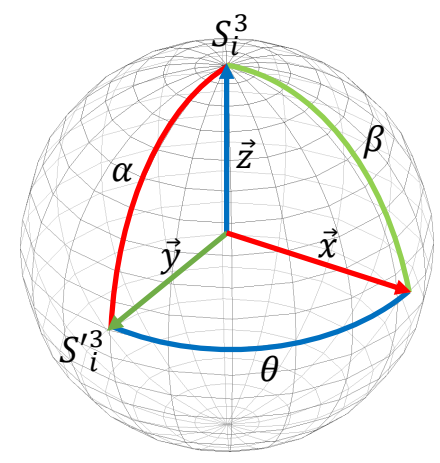

Fig. 3: Combination of rotations $\alpha, \beta$ and $\theta$ around $\vec{x}, \vec{y}$ and $\vec{z}$. In this case, it shows a single rotation around $\vec{x}$ of 90 [deg] from $S_{i}^{3}$ is at $(0,0,1)$ and that goes to $S_{i}^{\prime 3}$ at $(0,1,0)$.

Consequently, any combination of three rotations $(\alpha, \beta, \theta)$ around the 3 axis $\vec{x}, \vec{y}$ and $\vec{z}$ can be written as:

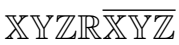

where $\mathbb{X}=\llbracket \cos (\alpha / 2), \vec{x} \sin (\alpha / 2) \rrbracket$

$$
\begin{aligned}
& \mathbb{Y}=\llbracket \cos (\beta / 2), \vec{y} \sin (\beta / 2) \rrbracket \\
& \mathbb{Z}=\llbracket \cos (\theta / 2), \vec{z} \sin (\theta / 2) \rrbracket
\end{aligned}
$$

The hypersphere $S^{3}$ [15] centered at the origin of the joint, can represent any configuration space $M^{3}$ of a rigidbody orientation without singularities [16] and avoid the addition of an extra reference frame. Our quaternions $\mathbb{X}, \mathbb{Y}$, and $\mathbb{Z}$ can be considered as points lying from $S_{i}^{3}$ to $S_{i}^{\prime}{ }^{3}$, as shown in Fig. 3.

\section{Hybrid Model DHKK-SRQ}

This part presents our new hybrid model DHKK-SQR that unifies the DHKK parameters with multiple sets of quaternions using an optimal analysis of the available sensor feedback. This unified formulation allows to express the kinematic of soft robots and reduces the corresponding computational cost.

Consequently, each $i-t h$ joint (for $i=1, \ldots, n$ where $n$ is the number of joints) is considered as an element that has a hybrid (rigid and soft) behavior. In Fig. 4, the $i-t h$ joint is modeled, in a first step as a rigid element, and only with the rotation $\theta_{i}$ around $\overrightarrow{z_{i}}$; finally, the rotations $\alpha_{i}$ and $\beta_{i}$ around axis $\overrightarrow{x_{i}}$ and $\overrightarrow{y_{i}}$ are added with the hypersphere $S_{i}^{3}$. As a result, the model can apply rotations in all axes avoiding to add any extra reference frames.

The rigid rotations are performed using the homogeneous matrices which are given by (1) and the kinematics of the rigid joints results from (2). The orientation and the final position are given by (3).

Using DHKK, the only possible active rotation is $\theta_{i}$ around the axes $\overrightarrow{z_{i}}$. Therefore, we formulate the extra rotations (those that appear from the low stiffness joints) $\alpha_{i}$ and $\beta_{i}$ using SRQ. Additionally, after the SRQ is launched, the rotation $\theta_{i}$ is formulated using a quaternion; so that, the set of quaternions for each $i-t h$ joint is like the proposed in (8).
To define when the SRQ is launched, avoiding the extra computational cost, our hybrid method is parameterized in three different cases depending on the available instrumentation: i) a fully instrumented setting, i.e. position and force sensors, ii) a partially instrumented robot with only position sensors (full or partial measure), and iii) no instrumentation available.

For the first case, the model use (3) to calculate the final position of the robot. Once the force sensor detects the contact with an object, we apply (9) to evaluate the absolute position error (Euclidian distance). If the error exceeds the threshold $\lambda$, the rotations are performed using (8), with the measured angles $\alpha=\left[\alpha_{1}, \ldots, \alpha_{n}\right], \beta=\left[\beta_{1}, \ldots, \beta_{n}\right]$ and $\theta=\left[\theta_{1}, \ldots, \theta_{n}\right]$ for the $n$ joints of the robot. The threshold $\lambda$ depends on the precision requirements of the robot.

$$
\sqrt{\sum_{x, y, z}\left|{ }^{0} P M_{n}-{ }^{0} P_{n}\right|^{2}} \leq \lambda
$$

Where, ${ }^{0} P M_{n}=\left[{ }^{0} P M_{n}^{x},{ }^{0} P M_{n}^{y},{ }^{0} P M_{n}^{z}\right]^{T}$ is the measured end position and ${ }^{0} P_{n}=\left[{ }^{0} P_{n}^{x},{ }^{0} P_{n}^{y},{ }^{0} P_{n}^{z}\right]^{T}$ is the calculated end position.

For the second case, the model use (3) to calculate the final position of the robot, and the criterion (9) is applied. Once the threshold $\lambda$ is overtaken, if the robot is instrumented to measure all parameters, the rotations are performed using (8), with the measured angles $\alpha=\left[\alpha_{1}, \ldots, \alpha_{n}\right], \beta=\left[\beta_{1}, \ldots, \beta_{n}\right]$ and $\theta=\left[\theta_{1}, \ldots, \theta_{n}\right]$. If the robot is equipped only with sensors for rigid joints, then we apply (10) to calculate angles $\alpha$ and $\beta$ of each joint, and the rotations are performed using (8).

$$
(\alpha, \beta)=\underset{\alpha, \beta}{\arg \min }\left(\sqrt{\sum_{x, y, z}\left|{ }^{0} P M_{n}-{ }^{0} P_{n}\right|^{2}}\right)
$$

The last case is proposed for robotic hand applications, where the grasping parameters (size, shape and weight of the object) are known, but the robot doesn't have sensory feedback. So, the known grasping information is used to parameterize our method.

To grasp in a steady way an object, it is necessary to consider four conditions [17]:

1) The Summatory of the applied forces and torques must be equal to zero.

2) The direction of the forces must be orthogonal to the tangential plane of the point of contact with the object, as shown in Fig. 5a. The maximal angle of the force $\varphi$ depends on the friction coefficient $\mu$ of the object (cone of force).

3) The center of application of forces $C_{N}$ must be as close as possible to the center of mass of the object $C$, as shown in Fig. $5 b$.

4) The area formed by the points where the force is applied $\Delta_{F}$, see Fig. $5 \mathrm{c}$, should be up to $\xi_{o}$, which is a constant defined as a function of the maximal distance between the point of application of force. 

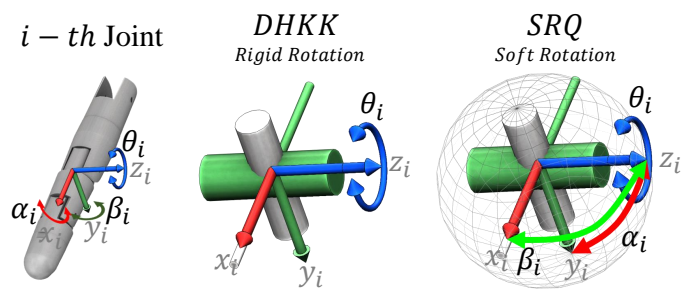

Fig. 4: Model of hybrid joint using DHKK and SRQ.

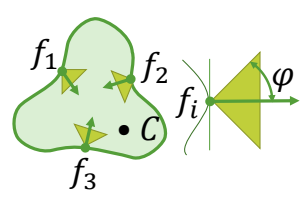

(a)

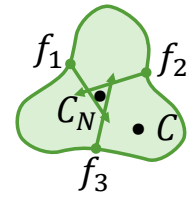

(b)

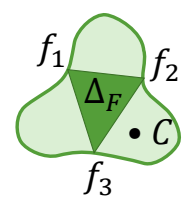

(c)
Fig. 5: Grasp Conditions.

These four conditions, can be expressed as follows:

$$
\begin{gathered}
\sum_{i} f_{i}=0 \text { and } \sum_{i} \tau_{i}=0 \\
\varphi=\arctan (\mu) \\
\left|C_{N}-C\right| \rightarrow \varepsilon \\
\Delta_{F} \rightarrow \xi_{o}
\end{gathered}
$$

where, $f_{i}$ and $\tau_{i}$ are the applied forces and torques with $i$ fingers and $\varepsilon$ is the maximal difference between the center of application of forces and the center of mass of the object.

On the basis of the above, we define the following criterion, to calculate the articular values required to position the robot in the required grasp posture as follows:

$$
\begin{gathered}
(\alpha, \beta, \theta)=\underset{\alpha, \beta, \theta}{\arg \max }\left(\Delta_{F}\right) \\
\text { s.t }\left|C_{N}-C\right|<\varepsilon, \Delta_{F}<\xi_{o}
\end{gathered}
$$

The rotations are performed using (1), while the values of $\alpha$ or $\beta$ are constant; then the rotations are formulated using SRQ as proposed in (8).

\section{Modeling of the Robotic Hand Prosthesis Promain-I}

The aim of the ProMain-I robotic hand is to perform precision grasping. Bearing in mind that the majority of this kind of grasping is achieved with only three fingers, our first approach is a three fingered bio-inspired hand. The hand is composed of two rigid joints in the wrist, and three hybrid joints in each finger. The joints metacarpophalangeal, proximal and distal interphalangeal are considered rigid for the flexion-extension movement and soft for the other rotations.

The hand is under-actuated, so each finger is controlled by only one servomotor. The equivalent mechanical model of the hand is shown in Fig. 6. The DHKK parameters for all

\begin{tabular}{|c|c|c|c|c|c|c|c|c|c|c|c|c|}
\hline \multirow{2}{*}{ Joint } & \multicolumn{12}{|c|}{ Wrist } \\
\hline & \multicolumn{4}{|c|}{$\alpha$} & \multicolumn{2}{|c|}{$a$} & \multicolumn{3}{|c|}{$d$} & \multicolumn{3}{|c|}{$\theta$} \\
\hline 1 & \multicolumn{3}{|c|}{0} & & \multicolumn{2}{|l|}{0} & \multicolumn{3}{|c|}{0} & \multicolumn{3}{|c|}{$\theta_{1}$} \\
\hline \multirow[t]{3}{*}{2} & & $-\frac{\pi}{2}$ & & & 0 & & & 0 & & & $\theta_{2}$ & \\
\hline & \multicolumn{4}{|c|}{ Middle Finger } & \multicolumn{4}{|c|}{ Index Finger } & \multicolumn{4}{|c|}{ Thumb Finger } \\
\hline & $\alpha$ & $a$ & $d$ & $\theta$ & $\alpha$ & $a$ & $d$ & $\theta$ & $\alpha$ & $a$ & $d$ & $\theta$ \\
\hline 3 & & $l_{31}$ & 0 & $\theta_{33}$ & 0 & $l_{21 x}$ & $21 z$ & $\theta_{23}$ & $\begin{array}{l}0 \\
0\end{array}$ & $\begin{array}{l}l_{11 x} \\
l_{11 y}\end{array}$ & $\begin{array}{c}l_{11 z} \\
0\end{array}$ & $\begin{array}{r}\frac{\pi}{2} \\
-\frac{\pi}{2}\end{array}$ \\
\hline 4 & 0 & $l_{32}$ & 0 & $\theta_{34}$ & 0 & $l_{22}$ & 0 & $\theta_{24}$ & $\alpha_{o p p}$ & $l_{12}$ & 0 & $\theta_{14}$ \\
\hline 5 & 0 & $l_{33}$ & 0 & $\theta_{35}$ & 0 & $l_{23}$ & 0 & $\theta_{25}$ & 0 & $l_{13}$ & 0 & $\theta_{15}$ \\
\hline$n$ & 0 & $l_{34}$ & 0 & 0 & 0 & $l_{24}$ & 0 & 0 & 0 & $l_{14}$ & 0 & 0 \\
\hline
\end{tabular}
joints are presented in Table. I, where the joints parameters for the wrist are joints one and two, and the parameters for

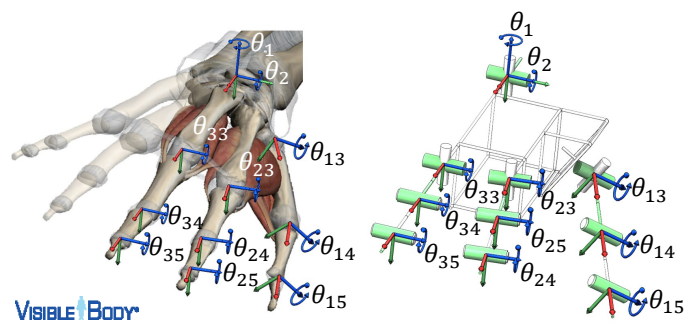

Fig. 6: Equivalent mechanical model of the hand, axes $x_{i}$ in red, axes $y_{i}$ in green and axes $z_{i}$ in blue $^{1}$

TABLE I: DHKK parameters for the ProMain-I Hand

the fingers are joints three to five. The parameters of fingers are denoted using a sub-index preceded by 1 for thumb finger, 2 for index finger and 3 for middle finger, e.g. for joint $i$ of the thumb we denotes the $\theta$ as $\theta_{1 i}$. The parameter $l_{31}$ is the distance between the wrist framework and the metacarpophalangeal joint of the middle finger. Parameters $l_{21 x}$ and $l_{21 z}$ are de distance from the wrist framework to the metacarpophalangeal joint of index finger. $l_{11 x}, l_{11 y}$ and $l_{11 z}$ represent the distance between the wrist framework and the carpometacarpal joint of thumb finger. Parameter $l_{22}, \ldots, l_{24}$ and $l_{22}, \ldots, l_{24}$ are the lengths of the index and the middle finger phalanges and $l_{12}, \ldots, l_{14}$ are the lengths of the metacarpal bone and the phalanges of the thumb finger.

Applying (1), for the parameters $\left[\alpha_{1 i}, a_{1 i}, d_{1 i}, \theta_{1 i}\right]$, $\left[\alpha_{2 i}, a_{2 i}, d_{2 i}, \theta_{2 i}\right]$ and $\left[\alpha_{3 i}, a_{3 i}, d_{3 i}, \theta_{3 i}\right]$, we obtain three rotation matrices (2) of the form ${ }^{0} T_{n}$, three orientation matrices of the form ${ }^{0} R_{n}$, and three position vectors of the form $\left[{ }^{0} P_{n}^{x},{ }^{0} P_{n}^{y},{ }^{0} P_{n}^{z}\right]^{T}$, that describe the end position of each finger.

On the other hand, to model soft rotation, we use the DHKK parameters $\left[\alpha_{j i}, a_{j i}, d_{j i}, \theta_{j i}\right]$, for the fingers $j=1, \ldots, 3$ and the joints $i=3, \ldots, 5$, to propose:

1) The set of vectors $\vec{r}_{j i}=d_{j i} \vec{x}_{j i}$ that match direction, orientation and length of the finger phalanges.

2) The set of pure quaternions $\mathbb{R}_{j i}=\llbracket 0, \vec{r}_{j i} \rrbracket$.

3) The sets of quaternions $\mathbb{X}_{j i}, \mathbb{Y}_{j i}, \mathbb{Z}_{j i}$, adding the rotations $\beta_{j i}$ around axes $y_{j i}$.

To formulate rotation as proposed in (8), we introduce the set of rotation for all fingers as follows:

\footnotetext{
${ }^{1}$ The hand image is courtesy of Visible Body (www.visiblebody.com)
} 


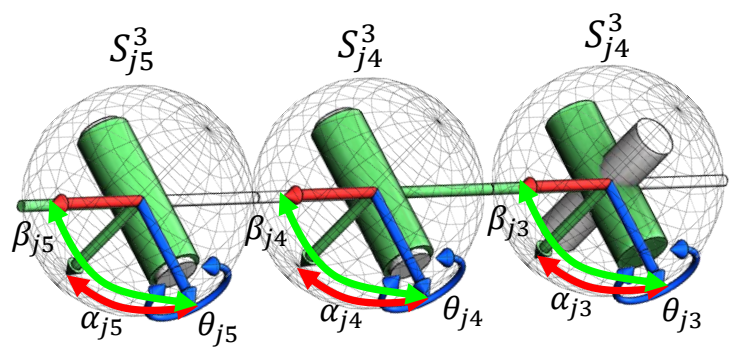

Fig. 7: Complete model of DHKK-SRQ for one finger $j$

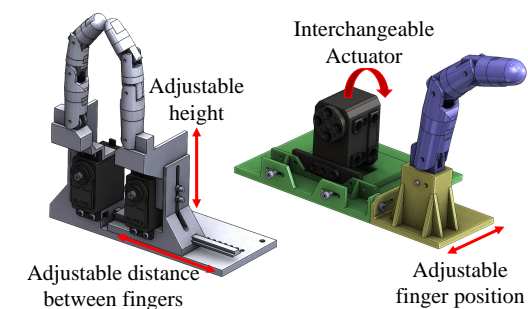

Fig. 8: CAD Model of the test platforms

$$
\mathbb{X}_{j i} \mathbb{Y}_{j i} \mathbb{Z}_{j i} \mathbb{R}_{j i} \overline{\mathbb{X}_{j i} \mathbb{Y}_{j i} \mathbb{Z}_{j i}}
$$

As a result, we have a model where one hypersphere $S_{j i}^{3}$ is added to each soft joint. In Fig. 7 the model DHKK-SRQ of one finger $j$ of the hand is shown.

\section{First Prototype of the Finger AND EXPERIMENTS}

In the first stage of the design and implementation of the ProMain-I Hand, we test a tendon driven finger prototype, which has three phalanges and is actuated by only one servomotor. The finger has rigid joints but due to the driving mechanism, it mechanically self-adjusts the articulation when is needed, this behavior can be considered as soft.

The aim of the test is to implement and try out our hybrid modeling method DHKK-SRQ and to obtain information to design the hybrid joints and drive mechanism of the ProMain-I Hand. Therefore, we design two platforms to carry out an experiment to measure the kinematics of the finger, and two fingers grip force and fingertip force. The experiment is performed for several servomotors and two configurations.

\section{A. Materials and methods}

The first platform is designed to place two fingers pointing toward each other, to perform a grip force between the fingers. The platform allows to set the distance between the fingers and the height to place several actuators. The second platform allows to change actuators and the position of the finger about the motor axis. The CAD models of the test platforms are shown in Fig. 8

To measure the force, we use a resistive-based force sensor Flexiforce $₫$, that measure up to $5 N$, connected to a circuit that uses an inverting operational amplifier arrangement to produce an analog output based on the sensor resistance,

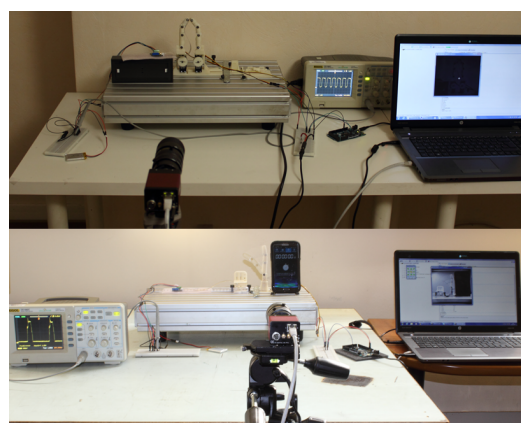

Fig. 9: Experiment Set-up

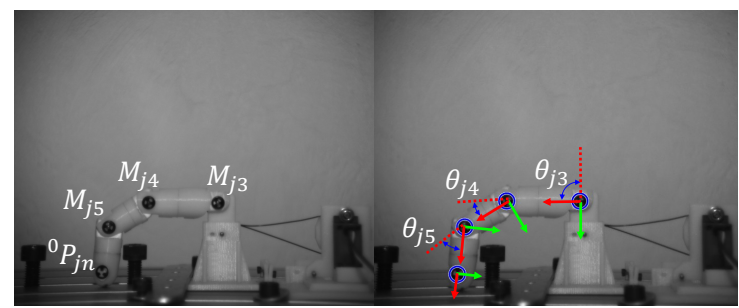

Fig. 10: Angles and final position measure

the output voltage is registered with a digital oscilloscope. The sensor was calibrated in the range $0.6 N$ to $4.8 N$. The sensor is placed on a support (platform) which is located in the trajectory of the fingertip.

Considering that the finger performs flexion and extension in $2 \mathrm{D}$, the kinematic was measured using a high-performance 4 megapixel CCD camera Prosilica GE-2040, to track black markers placed in the finger joints. The camera is placed at $1 \mathrm{~m}$ from the prototype; the optical objective is configured with an aperture of $F=1.4$, and a focal length of $20 \mathrm{~mm}$ and the shutter speed is $20 \mathrm{~ms}$. As the camera has a square format, the diameter limit of the circle of confusion is $C o C=0.053 \mathrm{~mm}$, which means that each pixel correspond to $0.17 \mathrm{~mm}$. After measure the precision of acquisition comparing several static images with a known value of length, we find a position accuracy of $0.51 \mathrm{~mm}$. The global setup of the experiment is shown in Fig. 9.

\section{B. Results}

The captured images are analyzed using Matlab ${ }^{\circledR}$ by applying a Canny filter and the Hough circular transform, to calculate the position of the fingertip and joint angles during the movement. As a result, we get a set of four points $\left(x_{i}, y_{i}\right)$. The first three points match with the position $M_{j} i$ of each $i$-th joint, and the last one is fingertip position ${ }^{0} P_{j n}$, where $j$ represents the finger. The angles are measured as shown in Fig. 10, following the DHKK parameterization.

The measured values are compared with the result of DHKK and DHKK-SRQ methods. To calculate the mean absolute error of position, the measures of the fingertip are compared with the calculated values, the results are shown in Table III. On the other hand, for the test of grip, the same analysis is performed but changing the distance between the 


\begin{tabular}{cccc}
\hline Distance & DHKK-Error & DHSKK-SRQ-Error & Grip Force \\
\hline $5.0 \mathrm{~cm}$ & $7.1193 \mathrm{~mm}$ & $7.0976 \mathrm{~mm}$ & $4.02 \mathrm{~N}$ \\
$5.5 \mathrm{~cm}$ & $7.1105 \mathrm{~mm}$ & $7.0906 \mathrm{~mm}$ & $4.60 \mathrm{~N}$ \\
$6.0 \mathrm{~cm}$ & $7.1193 \mathrm{~mm}$ & $7.097 \mathrm{~mm} 6$ & $4.70 \mathrm{~N}$ \\
$6.5 \mathrm{~cm}$ & $7.3092 \mathrm{~mm}$ & $7.1756 \mathrm{~mm}$ & $3.54 \mathrm{~N}$ \\
\hline
\end{tabular}

TABLE II: The mean absolute position error and grip force (2nd platform).

\begin{tabular}{cccc}
\hline Actuator & DHKK-Error & DHSKK-SRQ-Error & Force \\
\hline HS-422 & $2.0438 \mathrm{~mm}$ & $1.7927 \mathrm{~mm}$ & $2.19 \mathrm{~N}$ \\
TR-2065 & $0.6929 \mathrm{~mm}$ & $0.4206 \mathrm{~mm}$ & $1.19 \mathrm{~N}$ \\
Ax12a & $1.8032 \mathrm{~mm}$ & $1.3900 \mathrm{~mm}$ & $3.21 \mathrm{~N}$ \\
XL-320 & $2.2647 \mathrm{~mm}$ & $2.2300 \mathrm{~mm}$ & $2.10 \mathrm{~N}$ \\
MX-106R & $4.5700 \mathrm{~mm}$ & $4.4939 \mathrm{~mm}$ & $>5.0 \mathrm{~N}$ \\
\hline
\end{tabular}

TABLE III: The mean absolute position error and fingertip force (1st platform).

finger and using only one actuator (HS-422). The results of grip kinematics and force are presented in Table II.

\section{Discussion of Futures Developments}

Based on the obtained results, it is important to remark that our method presents a smaller error compared with the classical modeling of kinematics. Considering that the kinematic is measured in 2D, the effects of the angles $\alpha_{j i}$ and $\beta_{j i}$ are not taking into account, and so that, the error difference doesn't seem to be as big as expected. Even that, it is evident that this difference will become important for the final model of our ProMain-I hand and also for all soft robots.

Furthermore, the experiment of gripping, allow us to identify that the drive mechanism used for the first prototype needs to be improved, adding at less one soft degree of freedom in the metacarpophalangeal joint and introducing a fixed angle relationship between the sub-actuated joints and the actuator.

In this case, the force measure is used as a flag to launch the DHKK-SRQ, in future develops we plan to model the dynamic problem of the soft robots taking into account kinematics and forces.

\section{CONCLUSIONS}

In this work, we have proposed a new methodology to model the kinematic of any soft robots e.g. prostheses and exoskeletons. Experiments show that our model is more accurate than classical modeling of kinematics. Additionally, with the consideration of different utilization panoramas, the method can be set up to improve the computational cost. One critical application of soft robotics is in the medical domain and telesurgery, where the improvements of accuracy, provided by our method have a high impact. Our method also offers the possibility of new developments to improve the soft robotics in the next years. The advances of soft robotics could allow the design of robots more suitable for the interaction with humans, and consequentially, the robotic prostheses could be more acceptable to the patients. Finally, the consideration of the synergies simplifies the configuration space during the application of our DHKK-SRQ kinematic method.

\section{ACKNOWLEDGMENT}

We express our gratitude to the Université Paris Lumières for the financial support through the project PROMAIN This work has been supported by Université Paris Lumières and by a Scientific Mission funding from LEME-UPO-EA4416 / LIASD-UP8-EA4383. We acknowledge Colciencias and the UMNG for the financial support of the Ph.D. students.

\section{REFERENCES}

[1] S. Zodey and S. K. Pradhan, "Matlab Toolbox for Kinematic Analysis and Simulation of Dexterous Robotic Grippers," Procedia Engineering, vol. 97, pp. 1886-1895, 2014.

[2] C. Melchiorri, G. Palli, G. Berselli, and G. Vassura, "Development of the UB Hand IV: Overview of Design Solutions and Enabling Technologies," IEEE Robotics \& Automation Magazine, vol. 20, no. 3, pp. 72-81, Sept. 2013.

[3] J. T. Belter and A. M. Dollar, "Performance characteristics of anthropomorphic prosthetic hands," in IEEE International Conference on Rehabilitation Robotics, 2011, pp. 921-927.

[4] F. Ficuciello, G. Palli, C. Melchiorri, and B. Siciliano, "Experimental evaluation of the UB Hand IV postural synergies," in IEEE/RSJ International Conference on Intelligent Robots and Systems (IROS), San Francisco, CA, Sept. 2011, pp. 1775 - 1780.

[5] M. G. Catalano, G. Grioli, E. Farnioli, A. Serio, C. Piazza, and A. Bicchi, "Adaptive synergies for the design and control of the Pisa/IIT SoftHand," The International Journal of Robotics Research, vol. 33, no. 5, pp. 768-782, 2014.

[6] M. Gabiccini, A. Bicchi, Do. Prattichizzo, and M. Malvezzi, "On the role of hand synergies in the optimal choice of grasping forces," Autonomous Robots, vol. 31, no. 2-3, pp. 235-252, 2011.

[7] R. Campa, K. Camarillo, and L. Arias, "Kinematic modeling and control of robot manipulators via unit quaternions: Application to a spherical wrist," in 45th IEEE Conference on Decision and Control, San Diego, CA, USA, Dec. 2006, pp. 6474-6479.

[8] O. Heidari, H. M. Daniali, and S. M. Varedi, "Geometric design of 3r manipulators for three precision poses using dual quaternions," in Second RSI/ISM International Conference on Robotics and Mechatronics (ICRoM), Tehran, Iran, 2014, pp. 601-606.

[9] K. Harada and T. Anzai, "Multiple sweeping using quaternion operations," Computer-Aided Design, vol. 34, no. 11, pp. 815-822, 2002.

[10] L. Radavelli, R. Simoni, E. R. De Pieri, and D. Martins, "A comparative Study of the Kinematics of Robots Manipulators by Denavit-Hartenberg and Dual Quaternion," Mecnica Computacional, vol. 31, no. 15, pp. 2833-2848, Nov. 2012.

[11] V. Hugel and N. Jouandeau, "Automatic generation of humanoids geometric model parameters," in RoboCup 2013: Robot World Cup XVII, pp. 408-419. Springer, 2014.

[12] J. S. Dai, "An historical review of the theoretical development of rigid body displacements from Rodrigues parameters to the finite twist," Mechanism and Machine Theory, vol. 41, no. 1, pp. 41-52, Jan. 2006.

[13] S. L. Altmann, "Hamilton, rodrigues, and the quaternion scandal," Mathematics Magazine, vol. 62, no. 5, pp. 291-308, Dec. 1989.

[14] A. Perez and J. M. McCarthy, "Geometric design of RRP, RPR and PRR serial chains," Mechanism and Machine Theory, vol. 40, no. 11, pp. 1294-1311, Nov. 2005.

[15] L. Yan and L. Xu, "Study of diagonalization on skew self-conjugate matrix in quaternion field," in Second International Conference on Computational Intelligence and Natural Computing Proceedings (CINC), Wuhan, China, 2010, vol. 1, pp. 72-75.

[16] J. Yang and Y. Altintas, "Generalized kinematics of five-axis serial machines with non-singular tool path generation," International Journal of Machine Tools and Manufacture, vol. 75, pp. 119-132, Dec. 2013.

[17] T. Mishra, P. Guha, A. Dutta, and K. S. Venkatesh, "Stochastic re-grasp planning for vision aided capture of deforming and moving object," Mechatronics, vol. 19, no. 4, pp. 510 - 519, 2009. 